High-temperature superconductors

\section{Universal nodal Fermi velocity}

he mechanism that causes high-temperature superconductivity in copper oxide materials (cuprates) is still unknown, more than 15 years after it was discovered ${ }^{1}$. As the charge carriers (electrons or holes) are introduced into the parent antiferromagnetic insulator, a process called doping, the material evolves from an insulator to a superconductor, and eventually to a normal metal. This marked change of physical properties with doping ${ }^{2-7}$ indicates that doping dependence (non-universality) might be a general feature of these materials, but we find that, on the contrary, the low-energy Fermi velocity of electrons is in fact universal, even among different superconductor families.

We used high-resolution angle-resolved photoemission, which can directly probe how electrons move in materials ${ }^{8}$, to investigate hole-doped materials at various dopings in five different families of cuprates. These included $\left(\mathrm{La}_{2-x} \mathrm{Sr}_{x}\right) \mathrm{CuO}_{4}$ (LSCO) and $\left(\mathrm{La}_{2-x-y} \mathrm{Nd}_{y} \mathrm{Sr}_{x}\right) \mathrm{CuO}_{4}$ (Nd-LSCO), $\mathrm{Bi}_{2} \mathrm{Sr}_{2} \mathrm{CaCu}_{2} \mathrm{O}_{8} \quad$ (Bi2212), $\quad \mathrm{Bi}_{2} \mathrm{Sr}_{2} \mathrm{CuO}_{6}$ (Bi2201), $\left(\mathrm{Ca}_{2-x} \mathrm{Na}_{x}\right) \mathrm{CuO}_{2} \mathrm{Cl}_{2}$ (Na-CCOC) and $\mathrm{Tl}_{2} \mathrm{Ba}_{2} \mathrm{CuO}_{6}(\mathrm{Tl} 2201)$. The LSCO system in particular covers the entire doping range $(0<x \leqslant 0.3)$ over which the physical properties vary from insulator $(0 \leqslant x<0.03)$ to superconductors $(0.05<x<0.25)$ to overdoped non-superconducting metal $(x>0.25)$. Apart from the Na-CCOC data taken at the Stanford Synchrotron Radiation Laboratory, all samples were measured at the Advanced Light Source at Lawrence Berkeley National Laboratory (experimental details are presented elsewhere $\left.{ }^{9,10}\right)$.

Figure 1a shows the energy-momentum (dispersion) curves of the LSCO system with various dopings $(0<x \leqslant 0.3)$, measured along the $(0,0)-(\pi, \pi)$ direction in the Brillouin zone in reciprocal space. This diagonal direction is special in these superconductors because the anisotropic superconducting $\operatorname{gap}^{11}$, as well as the normal-state pseudogap ${ }^{12,13}$, is zero along this so-called nodal direction. Figure la shows that, for all dopings, there is a kink at an energy of about $70 \mathrm{meV}$ that separates the dispersion into a high-energy part (that is, further from the Fermi energy) and a low- energy part (that is, closer to the Fermi energy) with different slopes. Whereas the high-energy dispersion varies with doping, the dispersion converges within about $50 \mathrm{meV}$ of the Fermi energy, revealing a behaviour that is independent of doping. Correspondingly, a decrease is seen in the electron-scattering rate at an energy of about $70 \mathrm{meV}$, as indicated in Fig. $1 \mathrm{~b}$ for the LSCO $(x=0.063)$ sample.

The electron velocity can be extracted quantitatively from the slope in dispersion, as $v=\partial \epsilon / \partial k$. We have obtained the lowenergy velocity (the Fermi velocity) and the high-energy velocity as a function of doping for all five families of materials (see supplementary information). The Fermi velocity is nearly constant for all materials and dopings within an experimental error of about $20 \%$. In contrast, the high-energy velocity varies strongly with doping.

This invariance of nodal Fermi velocity in cuprates is surprising, given the range

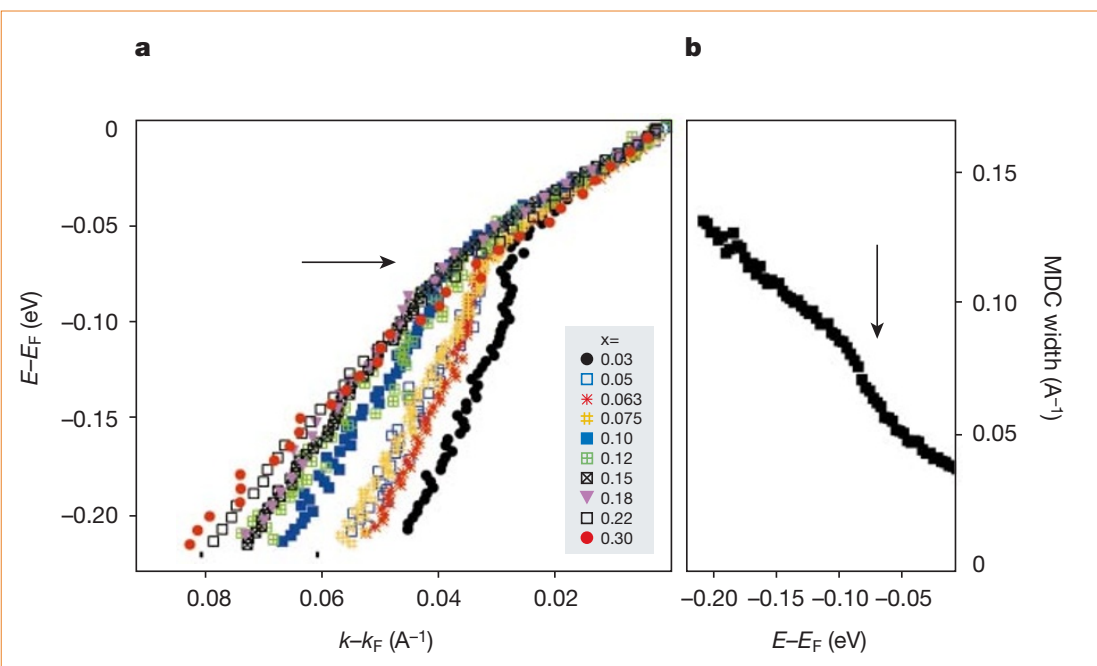

Figure 1 Electron dynamics in the $\left(\mathrm{La}_{2-x} \mathrm{Sr}_{x}\right) \mathrm{CuO}_{4}$ (LSCO) system. a, Dispersion energy, $E$, as a function of momentum, $k$, of LSCO with various dopings (where $x$ is between 0.03 (black circles, right curve) and about 0.30 (red circles, left) measured at a temperature of $20 \mathrm{~K}$ along the $(0,0)-(\pi, \pi)$ nodal direction. The dispersion is obtained by fitting momentum-distribution curves (MDCs), which represent the photoelectron intensity as a function of momentum, for a given energy. The arrow indicates the position of the kink that separates the dispersion into high-energy and low-energy parts with different slopes. $E_{\mathrm{F}}$ and $k_{\mathrm{F}}$, Fermi energy and Fermi momentum, respectively. b, Scattering rate as measured by MDC width (full width at half-maximum) of the LSCO $(x=0.063)$ sample measured at $20 \mathrm{~K}$. The MDC width is proportionally related to the scattering rate of electrons. The arrow indicates a decrease at an energy of about $70 \mathrm{meV}$. of variation in many other physical properties $^{2-7}$. This universal behaviour, together with the ubiquitous existence of a kink in the dispersion and a decrease in the scattering rate, are puzzles that require answers before the mystery of high-temperature superconductivity can be solved.

X. J. Zhou ${ }^{1,2}$ et al.*

e-mail:xjzhou@lbl.gov

${ }^{\star}$ T. Yoshida ${ }^{1,3}$, A. Lanzara ${ }^{1,2}$, P. V. Bogdanov ${ }^{1}$, S. A. Kellar ${ }^{1}$,

K. M. Shen ${ }^{1}$, W. L. Yang ${ }^{1,2}$, F. Ronning ${ }^{1}$, T. Sasagawa ${ }^{1}$,

T. Kakeshita ${ }^{4}$, T. Noda ${ }^{4}$, H. Eisaki ${ }^{4}$, S. Uchida ${ }^{4}$, C. T. Lin ${ }^{5}$,

F. Zhou ${ }^{6}$, J. W. Xiong ${ }^{6}$, W. X. Ti ${ }^{6}$, Z. X. Zhao ${ }^{6}$, A. Fujimori ${ }^{3}$,

Z. Hussain ${ }^{2}$ Z.-X. Shen

${ }^{1}$ Department of Physics, Applied Physics and SSRL, Stanford

University, Stanford, California 94305, USA ${ }^{2}$ Advanced Light

Source, Lawrence Berkeley National Laboratory, Berkeley,

California 94720, USA; Departments of ${ }^{3}$ Physics and

${ }^{4}$ Superconductivity, University of Tokyo, Bunkyo-ku, Tokyo 113,

Japan; ${ }^{5}$ Max-Planck-Institut für Festkörperforschung, 70569 Stuttgart, Germany; ${ }^{6}$ National Laboratory for Superconductivity, Institute of

Physics, Chinese Academy of Sciences, Beijing 100080, China.

1. Bednorz, J. G. \& Muller, K. A. Z. Phys. B 64, 189-193 (1986).

2. Ando, Y., Lavrov, A. N., Komiya, S., Segawa, K. \& Sun, X. F. Phys. Rev. Lett. 87, 17001-17004 (2001).

3. Uchida, S. et al. Phys. Rev. B 43, 7942-7954 (1991).

4. Yamada, K. et al. Phys. Rev. B 57, 6165-6172 (1998)

5. Loram, J. W., Luo, J. L., Cooper, J. R., Liang, W. Y. \& Tallon, J. L. Physica C 341-348, 831-834 (2000).

6. Uemura, Y. J. et al. Phys. Rev. Lett. 62, 2317-2320 (1989).

7. Timusk, T. \& Statt, B. Rep. Prog. Phys. 62, 61-122 (1999).

8. Huefner, S. Photoemission Spectroscopy: Principles and Applications (Springer, Berlin, 1995).

9. Zhou, X. J. et al. Phys. Rev. Lett. 86, 5578-5581 (2001).

10. Lanzara, A. et al. Nature 412, 510-514 (2001).

11. Shen, Z.-X. et al. Phys. Rev. Lett. 70, 1553-1556 (1993).

12. Loeser, A. G. et al. Science 273, 325-329 (1996).

13. Ding, H. et al. Nature 382, 51-54 (1996).

Supplementary information accompanies this communication on Nature's website.

Competing interests: declared none

Marine ecology

\section{Spring algal bloom and larval fish survival}

T he different factors that influence the prevalent decline in fish stocks are currently subject to urgent and intense scrutiny. Here we combine the use of remote-sensing satellite data with a longterm data set of haddock recruitment off the eastern continental shelf of Nova Scotia, Canada, to show that the survival of the larval fish depends on the timing of the local spring bloom of phytoplankton. This link has been suspected for more than 100 years, but its verification has had to wait for technology with sufficient spatial and temporal resolution.

A long-standing hypothesis ${ }^{1}$ contends that the abundance of fish year-classes is determined by food availability during the critical period of larval development. Variations in food supply between different years, probably determined by differences between the timing of the spring bloom of phytoplankton and the timing of fish spawning ${ }^{2-4}$, were thought to account for 
later variations in the abundance of adults (the Hjort-Cushing hypothesis).

It has not been feasible to test this possibility at the appropriate scales of time and space by using oceanographic research vessels. Nor was it previously possible to define objective criteria by which an ocean's pelagic ecosystem could be characterized on these scales $^{5}$ before the advent of remote-sensing by satellite of ocean colour, which reveals synoptic fields of phytoplankton biomass at a resolution of about $1 \mathrm{~km}$. Seasonal evolution of phytoplankton fields can be followed with a temporal resolution of 7 days by the construction of composite images. The spring bloom can therefore be characterized with respect to its peak amplitude, timing of peak, timing of initiation and duration.

We focus here on the timing of the bloom peak. A time series showing how this varies between years provides one element for testing the Hjort-Cushing hypothesis. For the other, we need to know how fish stocks varied in the same locality in the same years. From 1970 to the present, haddock (Melanogrammus aeglefinus) off the eastern Nova Scotian shelf have been surveyed annually after metamorphosis of the larvae; juveniles (0-2 years old) and adults were captured routinely in the survey. Juvenile abundance, normalized to spawning biomass or total egg production, provides an index of survival and can ultimately reveal the size of the incoming year-class.

For each of the 5,604 pixels $(1.5 \times 1.5$ $\mathrm{km}$ each) in the area where eggs and larvae are distributed (Fig. 1a), we determined the timing of the bloom peak in each year. The ocean-colour data cover the periods 1979-81 (CZCS sensor) and 1997-2001 (POLDER sensor for 1997 and SeaWiFS for 1998-2001). Before pooling the two data sets, we expressed each set as a vector of differences from the mean of the set, as a precaution against secular changes between the two data sets. We then tested the null hypothesis that variations in larval survival between years were independent of fluctuations in the timing of the spring phytoplankton bloom. We could account for $89 \%$ of the variance in larval survival by variation in the timing of the spring bloom (Fig. 1b). Our analysis is not compromised by differences between sensors or by any systematic error in the chlorophyllretrieval algorithms for any particular sensor, as we quantified only the timing of events.

Although our ocean-colour time series is short, the data indicate a link between bloom progression and larval survival. The data include two exceptional haddock year-classes (1981 and 1999; Fig. 1b) in the 32-year series covered by the groundfish survey; in both of these years, the spring
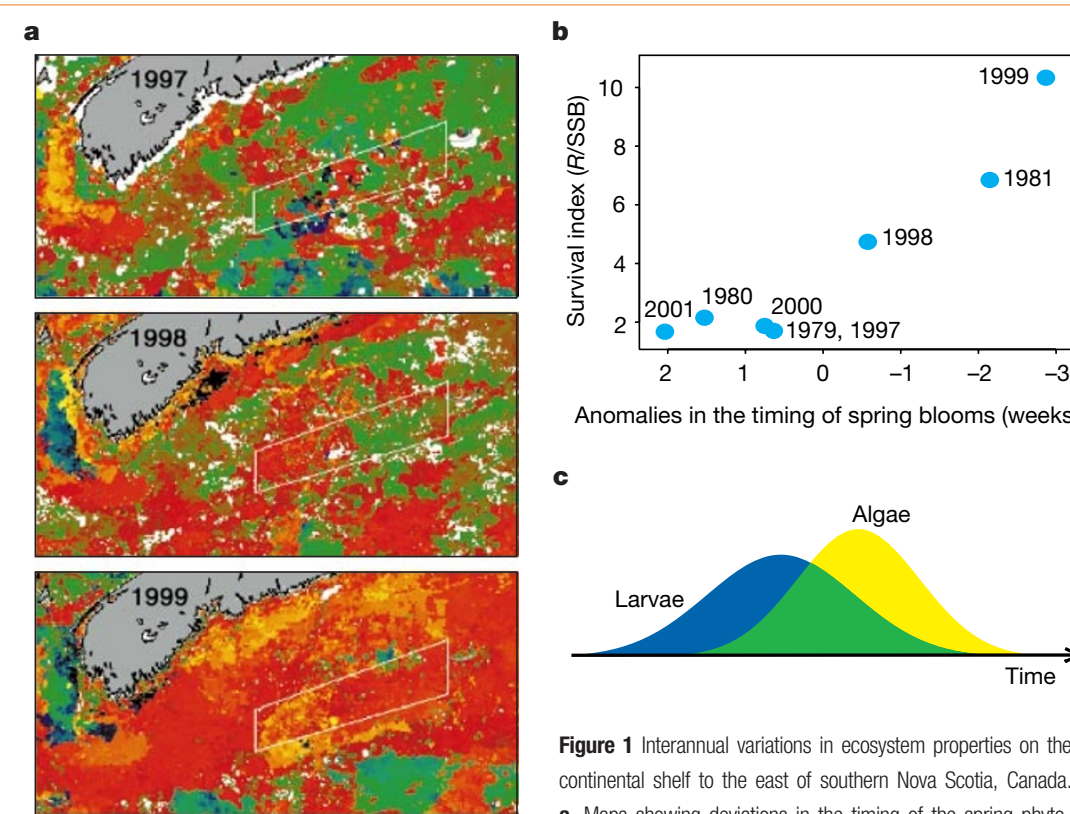

Anomalies in the timing of spring blooms (weeks)

c
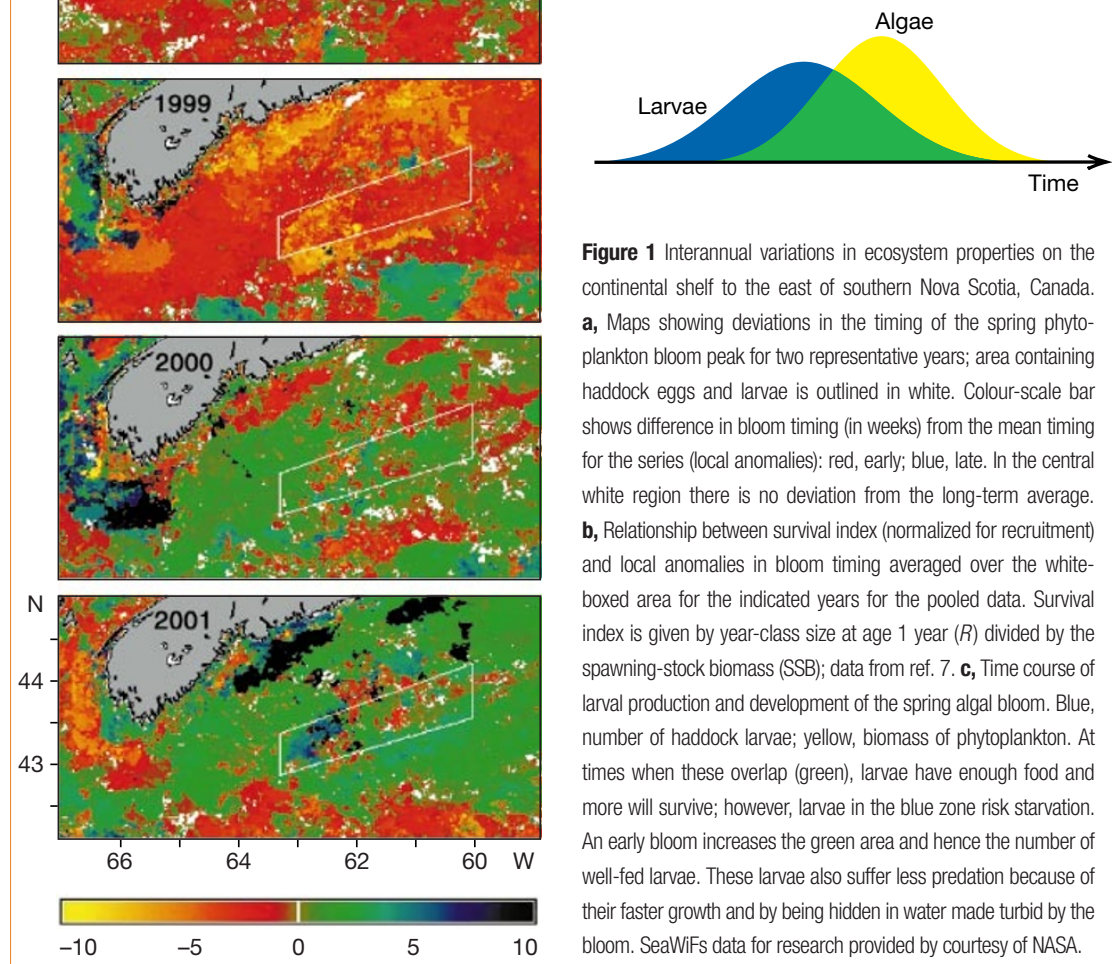

Figure 1 Interannual variations in ecosystem properties on the continental shelf to the east of southern Nova Scotia, Canada. a, Maps showing deviations in the timing of the spring phytoplankton bloom peak for two representative years; area containing haddock eggs and larvae is outlined in white. Colour-scale bar shows difference in bloom timing (in weeks) from the mean timing for the series (local anomalies): red, early; blue, late. In the central white region there is no deviation from the long-term average. b, Relationship between survival index (normalized for recruitment) and local anomalies in bloom timing averaged over the whiteboxed area for the indicated years for the pooled data. Survival index is given by year-class size at age 1 year $(R)$ divided by the spawning-stock biomass (SSB); data from ref. 7. c, Time course of larval production and development of the spring algal bloom. Blue, number of haddock larvae; yellow, biomass of phytoplankton. At times when these overlap (green), larvae have enough food and more will survive; however, larvae in the blue zone risk starvation. An early bloom increases the green area and hence the number of well-fed larvae. These larvae also suffer less predation because of their faster growth and by being hidden in water made turbid by the bloom. SeaWiFs data for research provided by courtesy of NASA.

blooms occurred unusually early. Some of the haddock population may therefore have begun to spawn early in the year, even though spawning peaked later. The advantage of an early bloom for a fish species with an extended spawning period might be that fewer of the total larvae produced perish from lack of food (Fig. 1c).

The mean and variance in spawning time presumably respond to environmental pressures in the broadest sense (including predation by humans). Although zooplankton dominate the diet of larval haddock, there is evidence that phytoplankton are also included ${ }^{6}$. Moreover, the Hjort-Cushing hypothesis refers explicitly to phytoplankton, rather than to zooplankton. Although an early spring phytoplankton bloom may not be sufficient to ensure high survival in the same year, it could be a necessary condition.

Our application of remotely sensed ocean-colour data has produced direct evidence for a putative trophic link that will be an important factor in future analyses of dwindling fish stocks, underlining the need to maintain a continuous, internally consistent stream of such data.

Trevor Platt ${ }^{\star}$, César Fuentes-Yaco ${ }^{\star} \dagger$, Kenneth T. Frank*

${ }^{*}$ Department of Fisheries and Oceans,

Bedford Institute of Oceanography, Ocean Sciences Division, Box 1006, Dartmouth, Nova Scotia B2Y 4A2, Canada

$\dagger$ Department of Oceanography, Dalhousie University, Halifax, Nova Scotia B3H 4J1, Canada e-mail:tplatt@dal.ca

1. Hjort, J. Rapp. Conserv. Explor. Mer 20, 1-228 (1914).

2. Cushing, D. H. in Sea Fisheries Research (ed. Harden Jones, F. R.) 399-412 (Elek Science, London, 1974).

3. Cushing, D. H. Adv. Mar. Biol. 26, 249-294 (1990).

4. Sverdrup, H. U. J. Conseil Perm. Int. Explor. Mer 18, 287-295 (1953).

5. Platt, T. \& Sathyendranath, S. Int. Coun. Explor. Sec CM O:3 (1996).

6. Kane, J. Mar. Ecol. Progr. Ser. 16, 9-20 (1984).

7. Mohn, R. K. \& Simon, J. E. CSAS Res. Doc. 2002/102 (2002). Competing financial interests: declared none. 